\title{
ASYMPTOTIC DISTRIBUTION OF LIUSTERNIK-SCHNIRELMAN EIGENVALUES FOR ELLIPTIC NONLINEAR OPERATORS
}

\author{
by V. B. MOSCATELLI and M. THOMPSON*
}

(Received 10th November 1988)

\begin{abstract}
Asymptotic formulae are given for the distribution of Liusternik-Schnirelman eigenvalues of certain pairs of nonlinear functionals generalising the usual Weyl theory for linear pairs of elliptic operators. In particular an application is made to the von Kármán theory of buckled plates.
\end{abstract}

1980 Mathematics subject classification (1985 Revision): 35P30, 35P20.

\section{Introduction}

Let $V$ be a reflexive Banach space and $\mathscr{A}(u), \mathscr{B}(u)$ two real valued $C^{1}$ functionals on $V$. For $c$ a real constant, set $M_{c}=\{u \in V: \mathscr{A}(u)=c\}$. Under certain technical conditions the variational theory of Liusternik-Schnirelman provides a natural generalisation of the Courant-Weinstein mini-max principle establishing the existence of an infinite number of distinct eigenpairs $\left(\lambda_{k}, u_{k}\right), u_{k} \in M_{c}, \lambda_{k} \in R$, of the Euler-Lagrange equations associated to the pair $(\mathscr{A}(u), \mathscr{B}(u))$ :

$$
A\left(u_{k}\right)=\lambda_{k} B\left(u_{k}\right)
$$

(see $[6,9])$.

In two papers $[7,8]$ Chiappinelli discussed the question of the determination of the properties of the asymptotic distribution of eigenvalues: $v(t)=\#\left\{k: \lambda_{k} \leqq t\right\}$. In the problems discussed in $[7,8],(\mathscr{A}, \mathscr{B})$ were chosen to be functionals related in $V=H_{0}^{1}(\Omega)$ to the pair of operators $(-\Delta u, u+f(u))$ and $(L u+f(x, u), u)$ respectively, where $f(u)$ satisfied a certain growth condition and $f(x, u)$ was taken to be sublinear and odd in $u$ with $L$ as a second order linear selfadjoint elliptic operator. Nevertheless lacunae were left and in the present paper we consider various variational problems in which we assume that $V$ is a closed subspace of the real Sobolev space $H^{m}(\Omega), \Omega$ a Sobolev domain in $R^{n}$ and $H_{0}^{m}(\Omega) \subset V, 2 m \geqq n$. Let $\mathscr{A}_{0}(u)$ and $\mathscr{B}_{0}(u)$ be the quadratic functionals associated with the self-adjoint linear differential operators in divergence from $A_{0}, B_{0}$ of

* Research supported by the CNPq under grant 300147-80. 
orders $2 m=2 m_{0}+2 m^{\prime}$ and $2 m^{\prime}$ respectively, $m_{0}>0, m^{\prime} \geqq 0$, whose coefficients are Hölder continuous of order $s, 0<s \leqq 1$, uniformly on $\bar{\Omega}$. Then more specifically we consider pairs of non-linear functionals $(\mathscr{A}(u), \mathscr{B}(u))$ taken in the form:

$$
\left(\frac{1}{2} \mathscr{A}_{0}(u)+f(u), \frac{1}{2} \mathscr{B}_{0}(u)+h(u)\right)
$$

where $f(u)$ and $h(u)$ are nonquadratic functionals satisfying certain technical conditions $\left(f(u)\right.$ satisfies essentially the conditions $(S)$ of [6] and $f, h$ are $C^{1}$ even potentials and $h^{\prime}(u)$ is compact in $V^{*}$, the normed conjugate of $V$, on bounded closed subsets of $V$ ).

We set $v^{0}(t)=\#\left\{k: \mu_{k} \leqq t ; A_{0} u_{k}^{0}=\mu_{k} B u_{k}^{0}\right\}$. It is known that $v^{0}(t)=g \delta t^{t / 2 m_{0}}+$ $O\left(t^{(n-s / s+1) / 2 m o}\right)$ as $t \rightarrow \infty$, where $\delta=|\Omega|$, the Lebesgue measure of $\Omega$, and $g$ is a geometric constant. Let

$$
j_{0}=\frac{2 m s}{s+1}\left(\frac{2 m s}{s+1}+m_{0}(2 m-n)\right)^{-1}<n / 2, \quad 2 m>n .
$$

In case $f \equiv 0$ and $h$ satisfies conditions $\mathrm{H} 3$, the corollary to Theorem 1 , states that for $2 m>n$, then setting $v(t)=\#\{k: \lambda \leqq t\}$, we have

$$
v(t)=g \delta t^{n / 2 m_{0}}+O\left(t^{(n-s / s+1) / 2 m_{0}}\right) \quad \text { as } t \rightarrow \infty, \quad \text { if } j_{0} \leqq j \leqq \frac{n}{2}
$$

and

$$
v(t)=g \delta t^{n / 2 m_{0}}+O\left(t^{n / 2 m_{0}-\sigma}\right) \quad \text { as } t \rightarrow \infty, \quad \text { in case } 0<j<j_{0},
$$

where

$$
\sigma=\frac{m-j}{n-2 j} \frac{n}{2 m}-\frac{1}{2}>0
$$

While in case $2 m=n$, we have merely that $v(t) \approx v^{0}(t)$ as $t \rightarrow \infty$ at level $M_{c}$. With the same conditions on $h$ but with $f(u)$ satisfying more general growth and coercivity conditions, we establish in Theorem 2 , that for $c$ sufficiently large $v(t) \approx v^{0}(t)$ as $t \rightarrow \infty$ at level $M_{c}$. Theorem 1 uses nonintegral interpolation estimates and Theorem 2 these together with the use of a radial odd homomorphism from $M_{c}$ to the level set $\Sigma_{c}=\left\{u: \mathscr{A}_{0}(u)=2 c\right\}$. We also give a result for the von Kármán equations describing plate buckling, taking advantage of the special form of the pairs, where $h \equiv 0$ and $f(u)$ is positive and compact on bounded closed subsets in $V=H_{0}^{2}(\Omega)$. Theorem 2 ' states that in this case $v(t)=g \delta t+O\left(t^{(2-s / s+1) / 2}\right)$ as $t \rightarrow \infty$ at level $M_{c}$. Here it should be noted that $f(u)$ is not a polynomial type expression in the derivatives of $u$ as it effectively involves pseudo-differential operators.

The first author (VBM) acknowledges the support of the Ministero della Publica Instruzione (Italy) and the second (MT) the support of ICTP (Trieste) and the CNPq (Brazil) while visiting Trieste and Lecce. 


\section{Preliminaries}

Let $\Omega$ be an open bounded region in $R^{n}$ with a sufficiently regular boundary (see [10] and the references given there). We denote the norm on the standard Sobolev spaces $H^{t, p}(\Omega)$ by \|\|$_{t, p}$, the $L^{p}$-norms by \|\|$_{p}$ and more briefly we denote $H^{2, p}(\Omega)$ by $H^{t}(\Omega)$. $H_{0}^{t}(\Omega)$ denotes the completion of $C_{0}^{\infty}(\Omega)$ in the norm \|\|$_{s, 2}$.

For two functions $\phi$ and $\psi$ defined on a common domain $D$ of some $R^{n}$ we write $\phi(y) \sim \psi(y)$ and $\phi(y) \approx \psi(y)$ to mean respectively that for $y \in D$ and $y \rightarrow \infty$ we have

$$
\phi(y)=\psi(y)(1+0(1)) \quad \text { and } \quad 0<a_{1} \leqq \frac{\phi(y)}{\psi(y)} \leqq a_{2}<\infty .
$$

We assume the following hypotheses.

H1(a) Let $V$ be a closed subspace of $H^{m}(\Omega)$ such that $H_{0}^{m}(\Omega) \subset V$.

We consider two formally symmetric strongly elliptic operators in divergence form $A_{0}, B_{0}$ of order $2 m=2 m_{0}+2 m^{\prime}$ and $2 m^{\prime}$ respectively, $m_{0}>0, m^{\prime} \geqq 0$ such that their associated bilinear forms $\mathscr{A}_{0}$ and $\mathscr{B}_{0}$ satisfy the following conditions with positive constants $c_{0}, b_{0}, \gamma$.

H1(b)

$$
\mathscr{A}_{0}(u, u)+\gamma\|u\|_{2}^{2} \geqq c_{0}\|u\|_{m, 2}^{2}, \quad u \in V .
$$

H1(c)

$$
\mathscr{B}_{0}(u, u) \geqq b_{0}\|u\|_{m^{\prime}, 2}^{2}, \quad u \in V
$$

H1(d) The coefficients of $A_{0}$ and $B_{0}$ are uniformly Hölder continuous of order $s$, $0<s \leqq 1$, on $\bar{\Omega}$.

We set $\mathscr{A}_{0}(u)=\mathscr{A}_{0}(u, u)$ and $\mathscr{B}_{0}(u)=\mathscr{B}_{0}(u, u)$. As remarked earlier we consider pairs of nonlinear even functionals $\mathscr{A}(u)=\frac{1}{2} \mathscr{A}_{0}(u)+f(u), \mathscr{B}(u)=\frac{1}{2} \mathscr{B}_{0}(u)+h(u), u \in V$. In Theorem 1 we deal with the degenerate case $f \equiv 0$ while in the case of the von Kármán equations $h(u) \equiv 0$. Both these cases may be placed within the context of the theory as formulated in Theorem 6.6.11 of [4], where by Propositions 5.2, 6.4 and Theorem 7 of [6] we may take both $\mathscr{A}$ and $\mathscr{B}$ to be $C^{1}$.

H2(a) $\mathscr{A}^{\prime}(u)$ is a $C^{1}$ odd gradient operator with $\mathscr{A}^{\prime}(0)=0$ and for any $u \neq 0, \mathscr{A}^{\prime}(s u) . u$ is a strictly increasing function of the positive real variable $s$.

$\mathrm{H}$ 2(b) The functional $\mathscr{A}(u)$ is coercive.

H2(c) When $u_{n} \rightarrow u$ weakly in $V$ and $\mathscr{A}^{\prime}\left(u_{n}\right)$ converges strongly in $V^{*}$ then $\mathscr{A}\left(u_{n}\right) \rightarrow \mathscr{A}(u)$ and $u_{n} \vec{s} u$ in $V$.

The last hypothesis stated there is replaced by the following in Sections 2, 3 and 4.1 taken together with $\mathrm{H} 1(\mathrm{c}, \mathrm{d})$. 
H3(a) Let $H(x, \xi)$ be a function defined on $\Omega \times R^{1}$, measurable in $x$ and $C^{1}$ in $\xi$ on $R^{1}$, for each fixed $x$ outside of a nullset in $\Omega$.

H3(b) $\partial_{\xi} H(x,-\xi)=-\partial_{\xi} H(x, \xi), x \in \Omega, \xi \in R^{1}$.

H3(c) $\partial_{\xi} H(x, \xi) \xi \geqq 0 ; x \in \Omega, \xi \in R^{1}$.

H3(d) $\left|\partial_{\xi} H(x, \xi)\right| \leqq c|\xi|^{q}, 1<q=n(n-2 j)^{-1}, x \in \Omega, \xi \in R^{1}$, where $0<j<n / 2$.

Then we define $h(u)$ by $h(u)=\int_{\Omega} H(x, u(x)) d x, u \in V$. These hypotheses lead to

H2(d) (1) $h(u)$ is well defined, bounded on bounded subsets and of class $C^{1}$ on $V$;

(2) $h^{\prime}(u)$ is compact on each bounded closed subset of $V$;

(3) $h(u)=\int_{0}^{1} h^{\prime}(s u) u d s$ is even and positive.

Note that these properties given in $\mathrm{H} 2$ (d) follow as in the Appendix to Section 1 of [5]. H2(d) gives the last hypothesis of Theorem 6.6.11 of [4].

In the case $f \equiv 0, \mathrm{H} 2$ is trivially satisfied, while for the von Kármán equations all the necessary verifications are given in Section 4.2 to establish (iv) of Theorem 6.6.11 of [4].

We consider the eigenvalue problem

$$
a(u, v)=\lambda b(u, v), \quad \text { for all } \quad v \in V,
$$

where $a(u, w)=\left(\mathscr{A}^{\prime}(u), v\right)$ and $b(u, v)=\left(\mathscr{B}^{\prime}(u), v\right)$. Let $M_{c}=\{u \in V: \mathscr{A}(u)=c\}$.

The Liusternik-Schnirelman max-min theory states that there exist an infinite number of distinct elements of $u_{k} \in M_{c}$ such that (1.1) is satisfied with a corresponding $\lambda_{k} \in R$. More specifically let $S_{k}=\left\{K \subset M_{c}: K\right.$ symmetric compact and cat $\left.K \geqq k\right\}$. Each eigenvalue pair $\left(\lambda_{k}, u_{k}\right)$ is associated with a critical level $c_{k}$ determined by

$$
c_{k}=\sup _{V \in S_{k}} \inf _{u \in V \subset M_{c}} \mathscr{B}(u)
$$

such that

$$
\mathscr{B}\left(u_{k}\right)=c_{k}
$$

and

$$
\lambda_{k}^{-1}=\frac{\left(\mathscr{B}^{\prime}\left(u_{k}\right) \cdot u_{k}\right)}{\left(\mathscr{A}^{\prime}\left(u_{k}\right) \cdot u_{k}\right)}
$$

(see $[6,4$, Chapter 6, 9]).

We expect that under reasonable conditions

$$
\lambda_{k}^{-1} \sim \frac{\mathscr{B}\left(u_{k}\right)}{2 c}=c^{-1} c_{k}
$$


Note that in case $f \equiv 0, h \equiv 0$, a quadratic pair, $M_{c}$ is the simpler level set $\Sigma_{c}=$ $\left\{u \in V: \frac{1}{2} \mathscr{A}_{0}(u)=c\right\}$.

Let us recall that the eigenvalues $\lambda_{k}^{0}$ of the linear problem $A_{0} u_{k}^{0}=\lambda_{k}^{0} B_{0} u_{k}^{0}$ are given by

$$
\lambda_{k}^{0}=(2 c)^{-1} \sup _{K \in S_{k}} \inf _{u \in K \subset \Sigma_{c}} \mathscr{B}_{0}(u)
$$

(see Lemma A 6, 7A of [4]).

It follows that our principal task is to show that the right hand sides of (1.5) and (1.6) are asymptotic as $k \rightarrow \infty$. It is useful to recall that the class $S_{k}$ is preserved under radial odd homeomorphisms $\tau$ of submanifolds of $V$ invariant under the involution $\pi: u \rightarrow-u$. This follows from Lemma $1.2 \mathrm{~d}$ of [6].

Although we maintain the same hypotheses as $h(u)$ in Section 4.1 the preceding formulation is not adequate to deal with the more general nonlinear functionals introduced in 4.1. However, in this case we note that the technical hypotheses on $f(u)$ are those of Theorem 10 of [6] while it is readily seen that under conditions $\mathrm{H} 3$ the underlying abstract elements of the theory given in Theorem 8 of [6] may be established and the results of Theorem 10 of [6] taken over without modification, namely those listed in equations (1.2) to (1.4).

We introduced the vector space $R^{s_{m}}$ whose elements are $\xi^{(m)}=\left\{\xi_{\alpha}:|\alpha| \leqq m\right\}$. Let $F\left(x, \xi^{(m)}\right)$ be the function defined on the trivial jet bundle $\Omega x R^{s_{m}} \rightarrow R^{1}$, which defines an operator on functions on $\Omega$, assigning to each $u$ another function $v$ on $\Omega$ with $v(x)=F\left(x, \xi^{(m)}(u)(x)\right), \quad x \in \Omega$, where $\xi^{(m)}(u)(x)=\left\{D^{\alpha} u(x),|\alpha| \leqq m\right\}$. Define the nonlinear functional $f(u)=\int_{\Omega} F\left(x, \xi^{(m)}(u)(x)\right) d x$.

We consider even functionals

$$
\mathscr{A}(u)=\frac{1}{2} \mathscr{A}_{0}(u)+f(u), \quad u \in V .
$$

Associated with the functional $\mathscr{A}(u)$ is the Euler-Lagrange operator

$$
A(u)=A_{0} u+\sum_{|\alpha| \leqq m}(-1)^{|\alpha|} D^{\alpha} F_{\xi_{\alpha}}\left(x, \xi^{(m)}(u)(x)\right)
$$

where $F_{\xi_{\alpha}}$ is the partial derivative of the function $F$ with respect to $\xi_{\alpha}$. Such functionals are well defined under hypotheses given in the Appendix to Section 1 of [5].

We refer to such hypotheses as being the "usual hypotheses" and observe that under these hypotheses the following properties hold (see [5] or [6]).

P1 $f(u)$ is well defined, bounded on bounded subsets and of class $C^{1}$ on $V$. For each $u, v \in V$

$$
f(u, v)=\sum_{|a| \leqq m}\left(F_{a}\left(\cdot, \xi^{(m)}(u), D^{\alpha} v\right)\right.
$$

is well defined and $f^{\prime}(u) \cdot v=f(u, v)$ for all $u, v \in V$. 
P2 $f^{\prime}(u)$ satisfies condition $(S)$ of Section 6, Definition 6.1 of [6], and is bounded on bounded subsets.

P3 $f^{\prime}(u) \cdot u \geqq c_{1}\|u\|_{m, 2}^{2}-c_{2}\|u\|_{m, 2}$, for all $u \in V$.

P4 There exists a continuous positive function $\psi_{1}(x), x \in R$, such that

$$
\left\{\begin{array}{l}
f^{\prime}(u) \cdot u \leqq \Psi_{1}\left(\|u\|_{m, 2}\right)\|u\|_{m, 2}=\Phi_{1}\left(\|u\|_{m, 2}\right) \\
f(u)
\end{array}\right.
$$

Note that the eigenvalue problem (1.1) is equivalent to

$$
\tilde{a}(u, v)=a(u, v)+b_{0}^{-1} \gamma b(u, v)=\left(\lambda+b_{0}^{-1} \gamma\right) b(u, v)
$$

for all $v \in V$, and so causes a shift in the eigenvalues $\lambda \rightarrow \lambda+b_{0}^{-1} \gamma$. As characteristically there exists a sequence of eigenvalues $\lambda_{n} \rightarrow \infty$ this will not make a difference to the asymptotic properties of $\lambda_{n}$ as $n \rightarrow \infty$. Note that H1(b,c) and P3 imply that

$$
\tilde{a}(u, u) \geqq\left(c_{0}+c_{1}\right)\|u\|_{m, 2}^{2}-c_{2}\|u\|_{m, 2} .
$$

From (1.7), (1.8) it follows without loss of generality we may assume that $\gamma=0$.

Let $\Gamma_{\delta_{0}}=\left\{u: u \in V ;\|u\|_{m, 2}^{2}=\delta_{0}\right\}$. From (1.8) it follows that there exists a $k>0$ such that for $\delta_{0} \geqq k$,

$$
\left(\mathscr{A}^{\prime}(u), u\right) \geqq \frac{\left(c_{0}+c_{1}\right)}{2} \delta_{0}
$$

Then as in [5], setting $u=r v,\|v\|_{m, 2}^{2}=k$

$$
\mathscr{A}(u)-\mathscr{A}(v)=\int_{1}^{r} \frac{\mathscr{A}^{\prime}(s v) s v d s}{s} \geqq \frac{\left(c_{0}+c_{1}\right)}{2} \log r, \quad r>1 .
$$

We conclude that

$$
\mathscr{A}(u) \geqq \frac{\left(c_{0}+c_{1}\right)}{2} \log \left(\frac{\delta_{0}}{k}\right)^{1 / 2}-\max _{\|v\|_{m, 2}^{2}=k}|\mathscr{A}(v)|>0,
$$

taking $\delta_{0}$ sufficiently large.

Note that (1.10a) implies that $\mathscr{A}(u) \rightarrow \infty$ with $\|u\|_{m, 2} \rightarrow \infty$. Also note that the hypotheses $\mathrm{H} 2$ (d) imply the existence of a constant $c_{4}>0$ such that

$$
\mathscr{A}_{0}(u) \leqq \frac{c_{4}}{2}\|u\|_{m, 2}^{2}+\Phi_{1}\left(\|u\|_{m, 2}\right)=\Phi\left(\|u\|_{m, 2}\right)
$$


Hence, if $\mathscr{A}(u)=c \rightarrow \infty$ it follows from (1.10b) that $\|u\|_{m, 2} \rightarrow \infty$. Therefore for $c$ sufficiently large $\|u\|_{m, 2} \geqq k$ so that $(1.10 \mathrm{a})$ is satisfied and we obtain the bound

$$
\|u\|_{m, 2} \leqq k \exp \left[2\left(c_{0}+c_{1}\right)^{-1}\left(c+\max _{\|v\|_{m, 2}^{2}=k}|\mathscr{A}(v)|\right)\right.
$$

$u \in M_{c}, c$ sufficiently large.

The following property holds:

P5 Under the hypotheses $\mathrm{H} 1, \mathrm{H} 3$ and the "usual" hypotheses on $f$, for $c$ sufficiently large, $\mathscr{B}(u)>0$ for $u \in M_{c}$, and given $d>0$, there exists $d_{1}>0$ such that for all $u \in M_{c}$ with $\mathscr{B}(u) \geqq d$ then $\mathscr{B}^{\prime}(u) \cdot u \geqq d_{1}>0$.

Now (1.10c) holds so that $\|u\|_{m, 2} \leqq$ const, $u \in M_{c}$, also by $H 2$ (d) it follows from the Appendix to Section 1 of [5] that $\mathscr{B}^{\prime}(u)$ is compact on closed bounded subsets of $V$. Suppose then there exists a sequence $u_{n} \in M_{c}, u_{n} \stackrel{\mathfrak{W}}{\rightarrow} u$, such that both $\mathscr{B}\left(u_{n}\right) \geqq d$ and $\left(\mathscr{B}^{\prime}\left(u_{n}\right) \cdot u_{n}\right) \rightarrow 0$. Then we conclude that $\mathscr{B}^{\prime}(u) \cdot u=0$ by compactness and weak convergence and by positivity $\mathscr{B}_{0}(u)=0$ so that $\|u\|_{m, 2}=0$ which implies $\mathscr{B}(u)=0$, while the potential formula implies that $\lim \mathscr{B}\left(u_{n}\right)=\mathscr{B}(u) \geqq d$ a contradiction.

The last property is proved in Lemma 3 and may be announced as follows:

P6 For $c$ sufficiently large each ray from the origin hits $M_{c}$ in exactly one point.

\section{A technical lemma}

Let us recall the Sobolev embedding

$$
H^{j}(\Omega) \subset L^{1}(\Omega), \quad \text { for } \quad 2 \leqq l \leqq 2 n(n-2 j)^{-1},
$$

valid for all $0<j<n / 2$ (see $[12, \S 4.6 .1]$ ). In the subsequent analysis, for definiteness, we set

$$
l=2 q=2 n(n-2 j)^{-1}
$$

Further recall the interpolation inequality in the $H^{s}(\Omega)$ Sobolev spaces:

$$
\|u\|_{j, 2} \leqq c(\Omega)\|u\|_{2}^{(m-j) / m}\|u\|_{m, 2}^{j / m} .
$$

This is a so-called multiplicative inequality familiar from interpolation theory (see [12, Remark 6, \$2.4.2]). For a proof we refer to [12, Theorem 1, \$4.3.1; Theorem 1, \$2.4.2 and formula $(v)$ of that theorem]. Note that the $W^{i, p}(\Omega)$ spaces defined in [12] coincide with $H^{i, p}(\Omega)$ in the case $p=2$.

We set 


$$
\theta=\frac{n}{m} \frac{m-j}{n-2 j}, \quad 0<j<\frac{n}{2}
$$

Then we have the following lemma.

Lemma 1. Under hypotheses $\mathrm{HI}, \mathrm{H} 3$ and properties $\mathrm{Pl}$ to $\mathrm{P} 4$ there exists a constant $K(\Omega, c)$ such that

$$
h(u) \leqq K(\Omega, c)\|u\|_{2}^{1+\theta}, \quad u \in M_{c}
$$

and

$$
h^{\prime}(u) \cdot u \leqq K(\Omega, c)\|u\|_{2}^{1+\theta}, \quad u \in M_{c},
$$

if $c$ is taken sufficiently large.

Proof. By hypotheses and the formula for potential operators we have $h^{\prime}(\tau u) \cdot u=$ $\int_{\Omega} u(x) H_{\xi}^{\prime}(x, \tau u(x)) d x \leqq C \tau^{q} \int_{\Omega}|u(x)|^{q+1} d x, 0 \leqq \tau \leqq 1$, and

$$
h(u) \leqq \frac{C}{q+1} \int_{\Omega}|u(x)|^{q+1} d x .
$$

From Schwartz's inequality it follows that

$$
h(u) \leqq \text { const }\|u\|_{s}^{\mid q}\|u\|_{2}
$$

Then using (2.1) and (2.2) we have

$$
\|u\|_{s} \leqq\|u\|_{j, 2} \leqq C(\Omega)\|u\|_{2}^{(m-j) / m}\|u\|_{m, 2}^{j / m}
$$

and from (2.4)

$$
h(u) \leqq \text { const }\|u\|_{2}^{1+(q(m-j) / m)}\|u\|_{m, 2}^{q j / m}
$$

Then (1.10c) yields the upper bound, if $c$ is taken sufficiently large,

$$
h(u) \leqq K(\Omega, c)\|u\|_{2}^{1+\theta},
$$

similar estimates give (2.3b).

Note that in the case $f \equiv 0$ the value of $c$ is unrestricted. Recall that in case $2 m>n, u$ can be modified on a set of measure zero so that $u \in C^{0}(\bar{\Omega})$ and one has the well known interpolation inequality (see Lemma 13.2 of [1]):

$$
|u(x)| \leqq \gamma_{s}\|u\|_{m}^{n / 2 m}\|u\|_{2}^{1-n / 2 m}
$$


This leads to an estimate of the form

$$
h(u) \leqq \text { const }\|u\|_{2}^{(q+1)(1-(n / 2 m))}
$$

However, as

$$
1+\frac{n}{n-2 j} \frac{m-j}{m}>\frac{2 m-n}{2 m}+\frac{n}{n-2 j} \frac{m-(n / 2)}{m}=\left(1+\frac{n}{n-2 j}\right)\left(1-\frac{n}{2 m}\right)
$$

we see that in (2.3a) and (2.3b) we have attained a higher power of $\|u\|_{2}$ which is desirable in the asymptotic analysis as we shall subsequently. Also any fixed value of $q>1$ may be attained by choosing $j$ suitably.

\section{The distribution of eigenvalues $f \equiv 0,2 m \geqq n$}

In the subsequent analysis we $\delta=|\Omega|$, the Lebesgue measure of $\Omega$.

We recall a basic result which may be derived following the line of arguments given in $[10,11]$. Although the latter paper deals with much more general bilinear (matrix) pairs and accordingly does not give explicit expressions for the second term in the asymptotic formula for the distribution function, it is evident that the estimates for scalar bilinear pairs as considered here may be obtained as in [9]. Using (1.9), (1.3) and (1.4) it follows that

$$
c_{t}^{0}=c(g \delta)^{2 m_{0} / n} t^{-2 m_{0} / n}+O\left(t^{-\left(\left(2 m_{0} / n\right)+((s / s+1) / n)\right)}\right)
$$

We now establish a fundamental lemma. We set

$$
\sigma=\frac{m-j}{n-2 j} \frac{n}{2 m}-\frac{1}{2} \geqq 0 \quad \text { if } \quad 2 m \geqq n
$$

Lemma 2. Under hypotheses $H 1, H 2$ and with $f \equiv 0$, we have

$$
c_{t}=c_{t}^{0}+0\left(t^{-(2 m o / n)(1+\sigma)}\right) \quad \text { as } \quad t \rightarrow \infty
$$

Proof. By hypothesis $\frac{1}{2} \mathscr{B}_{0}(u) \leqq \mathscr{B}(u), u \in M_{c} \equiv \Sigma_{c}$, so that it follows immediately that

$$
c_{t}^{0} \leqq c_{t}
$$

For an upper bound we note that from Lemma 1 and H1(c) we have

$$
\sup _{K \in S_{\mathfrak{t}}} \inf _{u \in K \subset M_{c}} \mathscr{B}(u) \leqq \sup _{K \in S_{\mathfrak{t}}} \inf _{u \in K \subset M_{c}}\left(\mathscr{B}_{0}(u)\right)
$$


where $\phi(x)$ is the continuous positive increasing function

$$
\phi(x)=\frac{x}{2}+b_{0}^{-(1+\theta) / 2} K(\Omega, c) x^{1+\theta / 2} .
$$

Then by an observation of Chiappinelli in $[7, \S 3]$ we have

$$
\sup _{K \in S_{t}} \inf _{u \in K \subset M_{c}}\left(\mathscr{B}_{0}(u)\right) \leqq \phi\left[\sup _{K \in S_{t}} \inf _{u \in K \subset M_{c}} \mathscr{B}_{0}(u)\right]
$$

It follows that from (3.1), (3.2) and (3.4)

$$
c_{t}^{0} \leqq c_{t} \leqq c_{t}^{0}+O\left(t^{-\left(2 m_{0} / n\right)(1+\theta) / 2}\right)=c_{t}^{0}+O\left(t^{-\left(2 m_{0} / n\right)(1+\sigma)}\right) \quad \text { as } \quad t \rightarrow \infty
$$

Theorem 1. Suppose that $f \equiv 0,2 m \geqq n$, and hypotheses $H 1$ and $H 2$ are satisfied.Then $\lambda_{t}^{-1}=c c_{t}^{0}+O\left(t^{-\left(2 m_{0} / n\right)(1+\sigma)}\right)$ as $t \rightarrow \infty$ at level $M_{c}$.

Proof. Recall that from (1.3), (1.4) and $\left(\mathscr{A}^{\prime}\left(u_{t}\right) \cdot u_{t}\right)=2 c$ we have

$$
\lambda_{t}^{-1}-c^{-1} c_{t}=\left[\frac{1}{2} \mathscr{B}\left(u_{t}\right) \cdot u_{t}-\mathscr{B}\left(u_{t}\right)\right] c^{-1} .
$$

Set $W_{t}=\left(\frac{1}{2} h^{\prime}\left(u_{t}\right) \cdot u_{t}-h\left(u_{t}\right)\right) c^{-1}$. Then by inequalities $(2.3 \mathrm{a}, \mathrm{b})$ we have

$$
\left|W_{t}\right| \leqq \frac{3}{2} c^{-1} K(\Omega, c)\left\|u_{t}\right\|_{2}^{1+\theta} \leqq \frac{3}{2} c^{-1} K(\Omega, c) b_{0}^{-(1+\theta) / 2} \mathscr{B}_{0}\left(u_{t}\right)^{1+\theta / 2},
$$

using $\mathrm{H} 1(\mathrm{c})$,

$$
\begin{aligned}
& \leqq C_{1}(\Omega, c) \mathscr{B}\left(u_{t}\right)^{1+\theta / 2}, \quad \text { by } \quad \mathscr{B}_{0}(u) \leqq \mathscr{B}(u), \\
& =C_{1}(\Omega, c)\left(c_{t}\right)^{1+\theta / 2} \\
& \leqq C_{2}(\Omega, c) t^{-\left(2 m_{0} / n\right)(1+\sigma)}, \quad \text { using Lemma } 2 .
\end{aligned}
$$

From (3.5) and (3.6) we have

$$
\lambda_{t}^{-1}=c^{-1} c_{t}^{0}+0\left(t^{-\left(2 m_{0} / n\right)(1+\sigma)}\right) \quad \text { as } \quad t \rightarrow \infty .
$$

Corollary. Set

$$
j_{0}=\frac{2 m s}{s+1}\left(\frac{2 m s}{s+1}+m_{0}(2 m-n)\right)^{-1}
$$

in the case $2 m>n$

(a) If $j_{0} \leqq j \leqq n / 2$, then 


$$
v(t)=g \delta t^{n / 2 m_{0}}+O\left(t^{(n-(s / s+1)) / 2 m_{0}}\right) \quad \text { as } \quad t \rightarrow \infty \quad \text { at level } M_{c} .
$$

(b) If $0<j<j_{0}$, then

$$
v(t)=g \delta t^{n / 2 m_{0}}+0\left(t^{\left(n / 2 m_{0}\right)-\sigma}\right) \quad \text { as } \quad t \rightarrow \infty \quad \text { at level } M_{c}
$$

(c) In the case $2 m=n$, then

$$
v(t) \approx t^{n / 2 m_{0}} \quad \text { as } \quad t \rightarrow \infty \quad \text { at level } M_{c} .
$$

Proof. First note that since $m_{0}(2 m-n)>0$, in the case $2 m>n$, then $j_{0}<n / 2$.

We deal with cases (a) and (b). By (3.1) and Theorem 1 we have

$$
\lambda_{t}^{-1}=(g \delta)^{2 m 0 / n} t^{-(2 m 0 / n)}+O\left(E_{1}\right)+O\left(E_{2}\right) \quad \text { as } \quad t \rightarrow \infty .
$$

where $E_{1}=t^{-\left(2 m_{0}+(s / s+1)\right) / n}$ and $E_{2}=t^{-\left(2 m_{0} / n\right)(1+a)}$. It follows that $E_{1}$ or $E_{2}$ will be dominant in second order asymptotics depending as to whether

$$
2 m_{0} \sigma \gtreqless \frac{s}{s+1},
$$

or as to whether

$$
\frac{m_{0}(2 m-n) j}{m(n-2 j)} \gtreqless \frac{s}{s+1}
$$

leading to the inequalities $j \gtreqless j_{0}$. In the case $j_{0} \leqq j<n / 2$, (a) holds and, in the case, $0<j<j_{0}$ (b) holds.

Finally, if $2 m=n$, from (3.1) and Theorem 1 we see that

$$
\lambda_{t} \approx t^{2 m o / n}
$$

giving result (c).

\subsection{Estimates in the general case $2 m \geqq n$}

An important element in the theory of Liusternik-Schnirelman as given in [6, Theorems 10 and 8] is the property previously indicated, (P6). The following lemma establishes P6 in this case, the last property necessary to apply the theory of [6].

Lemma 3. Under conditions which guarantee properties P1, P2, P3, P4 (the "usual" hypotheses) together with $H I(b)$ and $H I(d)$ each ray from the origin hits $M_{c}$ in exactly one point if $c$ is taken to be sufficiently large. 
Proof. Let $u \in M_{c}$. If this property does not hold then there exists a positive $t \neq 0,1$ such that $u \in M_{c}$. Consider first the possibility that $0<t<1$. Then we have

$$
\frac{1}{2} t^{2} \mathscr{A}_{0}(u)+t \int_{0}^{1}\left(f^{\prime}(s t u) \cdot u\right) d s=c
$$

so that setting $\sigma^{\prime}=1-t^{2}$ and $s t=\tau$, we observe that

$$
\frac{1}{2}\left(1-\sigma^{\prime}\right) \mathscr{A}_{0}(u)+\int_{0}^{\sqrt{1-\sigma^{\prime}}}\left(f^{\prime}(\tau u) \cdot u\right) d \tau=c .
$$

Subtracting this from $\mathscr{A}(u)=c$, we see that

$$
\frac{1}{2} \sigma^{\prime} \mathscr{A}_{0}(u)+\int_{\sqrt{1-\sigma}}^{1} \frac{f^{\prime}(\tau u) \cdot \tau u}{\tau u} d \tau=0
$$

Using property $\mathrm{P} 3$, we conclude that

$$
f^{\prime}(\tau u) \cdot \tau u \geqq c_{1} \tau^{2}\|u\|_{m, 2}^{2}-c_{2} \tau\|u\|_{m, 2}
$$

and, hence, that

$$
0 \geqq \frac{1}{2} \sigma^{\prime} c_{0}\|u\|_{m, 2}^{2}+\int_{\sqrt{1}-\sigma^{\prime}}^{1}\left(c_{1} \tau\|u\|_{m, 2}^{2}-c_{2}\|u\|_{m, 2}\right) d \tau .
$$

Moreover, for $c$ sufficiently large $\|u\|_{m, 2} \geqq k>0$ from (1.10b) and we conclude that

$$
0 \geqq \frac{1}{2} \sigma^{\prime} c_{0}\|u\|_{m, 2}+c_{1}\|u\|_{m, 2}\left[\frac{\tau^{2}}{2}\right]_{\sqrt{1-\sigma^{\prime}}}^{1}-c_{2} \sigma^{\prime}[\tau]_{\sqrt{1-\sigma^{\prime}}}^{1}
$$

or

$$
0 \geqq \frac{1}{2} \sigma^{\prime}\left(c_{0}+c_{1}\right)\|u\|_{m, 2}-\frac{c_{2}}{1+\sqrt{1-\sigma^{\prime}}}
$$

However, from (1.10b), we have seen that $\|u\|_{m, 2} \rightarrow \infty$ as $c \rightarrow \infty, u \in M_{c}$. It follows that for $c$ sufficiently large to guarantee

$$
\|u\|_{m, 2}>\frac{2 c_{2}}{\left(c_{0}+c_{1}\right)\left(1+\sqrt{1-\sigma^{\prime}}\right)}
$$

the preceding inequality yields a contradiction, ruling out the possibility that $0<t<1$. The hypotheses that $t>1$ may be dealt with similarly, setting $\sigma^{\prime}=t^{2}-1$, with an argument which leads to the inequality 


$$
\frac{\left(c_{0}+c_{1}\right)}{2}\|u\|_{m, 2} \leqq \frac{c_{2}}{1+\sqrt{1+\sigma^{\prime}}}
$$

once again leading to contradiction for $c$ sufficiently large.

As an immediate consequence of Lemma 3 it follows that $\hat{\tau}(u)=(1-(f(u) / c))^{-1 / 2} u$ is an odd, radial, one-one onto map of $M_{c}$ to $\Sigma_{c}$, when $c$ is sufficiently large. We

$$
\operatorname{set} \tau(u)=(1-(f(u) / c))^{-1 / 2}=\sqrt{2 c} \mathscr{A}_{0}(u)^{-1 / 2}, u \in M_{c} .
$$

In fact, a simple modification of the argument given in Proposition 6.4 of [6] serves to show that $\hat{\tau}(u)$ is a homeomorphism (in fact, a diffeomorphism of the Finsler manifolds involved). In order to see this, renorm $V$ by the equivalent norm $\|u\| \|^{2}=$ $(2 c)^{-1} \mathscr{A}_{0}(u)$. This equivalence follows from $\mathrm{H} 1(\mathrm{~b})$, with $\gamma=0$ as we always suppose, together with $\mathscr{A}_{0}(u) \leqq c_{4}\|u\|_{m, 2}^{2}$, which follows from H1(d). Then $\Sigma_{c}$ becomes the unit ball in norm |||| $\mid$ and one may apply the result cited above.

Theorem 2. Assume that the hypotheses $\mathrm{H1}, \mathrm{H} 2$ hold together with the usual hypotheses on $f$ that $2 m>n$ and that $c$ is taken sufficiently large as in Lemma 3. Then we have $v(t) \approx t^{n / 2 m_{0}}$ as $t \rightarrow \infty$ at level $M_{c}$.

Proof. Under the conditions we have assumed all the conclusions of Theorem 10 of [6] are applicable. It follows that

$$
\begin{aligned}
& c_{t}=\sup _{V \in S_{t}} \inf _{u \in V \subset M_{c}}\left[\left(1-\frac{f(u)}{c}\right) \mathscr{B}_{0}(\hat{\tau}(u)) / 2\right. \\
& \left.+\int_{0}^{1} h^{\prime}\left(s\left(1-\frac{f(u)}{c}\right)^{1 / 2} \hat{\tau}(u)\right) \cdot\left(\left(1-\frac{f(u)}{c}\right)^{1 / 2} \hat{\tau}(u)\right) d s\right] \\
& \leqq \sup _{V \in S_{\boldsymbol{t}^{\prime}}} \inf _{\tilde{t}(u) \in \tilde{\tau}(V) \in \Sigma_{c}}\left(\frac{\mathscr{B}_{0}(\hat{\tau}(u))}{2}+\int_{0}^{(1-(S(u) / c))^{1 / 2}} h^{\prime}(s \hat{\tau}(u) \cdot \hat{\tau}(u)) d s\right) \\
& \leqq \sup _{V \in S_{t}} \inf _{u \in V \subset \Sigma_{c}}\left(\frac{\mathscr{B}_{0}(u)}{2}+\int_{0}^{1}\left(h^{\prime}(s u) \cdot u\right) d s\right) \\
& =\sup _{V \in S_{t}} \inf _{u \in V \subset \Sigma_{c}} \mathscr{B}(u)=\hat{c}_{t},
\end{aligned}
$$

by the invariance of the class $S_{t}$ under the odd radial homeomorphism $\hat{\tau}(u)$. However, Lemma 2 may be applied to $\hat{c}_{t}$ and we obtain 


$$
c_{t} \leqq c_{t}^{0}(1+O(1)) \quad \text { as } \quad t \rightarrow \infty
$$

For an inequality in the reverse direction observe that

$$
\begin{aligned}
\hat{\boldsymbol{c}}_{\mathbf{t}} & =\sup _{V \in S_{\mathfrak{t}}} \inf _{v \in V \in \Sigma_{c}} \mathscr{B}(v) \\
& =\sup _{V \in S_{t}} \inf _{u \in V \subset M_{c}}\left[\tau(u)^{2} \frac{\mathscr{B}_{0}(u)}{2}+\int_{0}^{1}\left(h^{\prime}(s \tau(u) u) \cdot \tau(u) u\right) d s\right],
\end{aligned}
$$

using the invariance of $S_{t}$ under the odd radial homeomorphism $\hat{\tau}^{-1}$,

$$
\begin{aligned}
\leqq \sup _{V \in S_{t}} \inf _{u \in V \subset M_{c}}[ & (2 c) \mathscr{A}_{0}(u)^{-1} \frac{\mathscr{B}_{0}(u)}{2} \\
& \left.+\frac{(2 c)^{(q+1) / 2} \mathscr{A}_{0}(u)^{-(q+1) / 2}}{q+1} K(\Omega, c)\|u\|_{2}^{1+\theta}\right],
\end{aligned}
$$

using Lemma 1, so that

$$
\hat{c}_{t} \leqq \text { const } \sup _{V \in S_{t}} \inf _{u \in V \subset M_{c}}\left[\|u\|_{m, 2}^{-2} \mathscr{B}(u)+\|u\|_{m, 2}^{-(q+1)} \mathscr{B}(u)^{(1+\theta) / 2}\right]
$$

using $\mathrm{H} 1(\mathrm{~b}), \mathrm{H} 1(\mathrm{c})$ and $\mathscr{B}_{0}(u) \leqq \mathscr{B}(u)$. By $(1.10 \mathrm{~b}),\|u\|_{m, 2} \geqq k$ for $c$ sufficiently large and hence we have

$$
\begin{aligned}
\hat{c}_{t} & \leqq \text { const } \sup _{V \in S_{t}} \inf _{u \in V \subset M_{c}}\left\{\mathscr{B}(u)+\mathscr{B}(u)^{(1+\theta) / 2}\right\} \\
& \leqq \operatorname{const}\left(c_{t}+\left(c_{t}\right)^{(1+\theta) / 2}\right) \\
& \leqq O(1) c_{t}^{0}+O\left(\left(c_{t}^{0}\right)^{(1+\theta) / 2}\right), \quad \text { as } t \rightarrow \infty
\end{aligned}
$$

using (4.1)

From (4.1) and (4.2) we conclude that 


$$
c_{t} \approx c_{t}^{0} \quad \text { as } \quad t \rightarrow \infty
$$

Recall that by (1.4)

$$
\lambda_{t}^{-1}=\frac{\left(\mathscr{B}^{\prime}\left(u_{t}\right) \cdot u_{t}\right)}{\left(\mathscr{A}^{\prime}\left(u_{t}\right) \cdot u_{t}\right)}, \quad u_{t} \in M_{c}
$$

By (1.9) we have for $\|u\|_{m, 2} \geqq k$,

$$
\left(\mathscr{A}^{\prime}(u) \cdot u\right) \geqq \frac{\left(c_{0}+c_{1}\right)}{2}\|u\|_{m, 2}^{2}
$$

and since by (1.10b), $\|u\|_{m, 2} \geqq k$ for $c$ sufficiently large

$$
\left(\mathscr{A}^{\prime}\left(u_{t}\right) \cdot u_{t}\right) \geqq \frac{\left(c_{0}+c_{1}\right)}{2} k^{2}
$$

Also from (1.10b) we have

$$
\left(\mathscr{A}^{\prime}\left(u_{t}\right) \cdot u_{t}\right) \leqq \Phi\left(\left\|u_{t}\right\|_{m, 2}\right)
$$

We conclude from (1.10c) and (4.6) that

$$
\left(\mathscr{A}^{\prime}\left(u_{t}\right) \cdot u_{t}\right) \leqq \Phi\left(k \exp 2\left(c_{0}+c_{1}\right)^{-1}\left[c+\max _{\|v\|_{m, 2}^{2}=k}|\mathscr{A}(v)|\right)\right.
$$

Now (4.5) and (4.7) imply that there exist positive constants $A_{1}$ and $A_{2}$ such that

$$
A_{1}\left(\mathscr{B}^{\prime}\left(u_{t}\right) \cdot u_{t}\right) \leqq \lambda_{t}^{-1} \leqq A_{2}\left(\mathscr{B}^{\prime}\left(u_{t}\right) \cdot u_{t}\right)
$$

As in Theorem 1 one shows that

$$
c^{-1}\left[\frac{1}{2}\left(\mathscr{B}{ }^{\prime}\left(u_{t}\right) \cdot u_{t}\right)-\mathscr{B}\left(u_{t}\right)\right]=W_{t}
$$

satisfies

$$
\left|W_{t}\right|=O\left(\left(c_{t}\right)^{(1+\theta) / 2}\right)
$$

Remembering $c_{t}=\mathscr{B}\left(u_{t}\right)$, by (1.3), and using (4.3), (4.8) and (4.9) we have established that $\lambda_{t}^{-1} \approx c_{t}^{0}$ as $t \rightarrow \infty$ at level $M_{c}$, for $c$ sufficiently large and the theorem is established. 


\subsection{The von Kármán equations for buckled plates}

In this special case the result given in Theorem 2 may be considerably refined.

It is convenient to introduce the notation

$$
[f, g]=\left(f_{y y} g_{x}-f_{x y} g_{y}\right)_{x}+\left(f_{x x} g_{y}-f_{x y} g_{x}\right)_{y}
$$

The von Kármán equations for deformations produced in a two dimensional elastic plate of shape $\Omega \subset R^{2}$ subject to compressive forces of magnitude $\lambda$ on its boundary $\partial \Omega$ may be written (see $[4, \S 1.1,2.5 \mathrm{c}, 6.2 \mathrm{~b}]$ ) in the form

$$
\begin{gathered}
\Delta^{2} u=\lambda[\Phi, u]+[u, \phi] \quad \text { in } \Omega \\
\Delta^{2} \phi=-[u, u] ; \\
u=u_{x}=u_{y}=0 \quad \text { on } \partial \Omega . \\
\phi=\phi_{x}=\phi_{y}=0
\end{gathered}
$$

In (4.10), (4.11) $\Phi+\lambda$ represents the Airy stress function and $u$ is the vertical deflection of the plate from its undeformed state.

$H^{\prime}$ (a) We assume that the matrix

$$
\left(\begin{array}{cc}
\partial_{x}^{2} \Phi & -\partial_{x y} \Phi \\
\partial_{x y} \Phi & \partial_{y}^{2} \Phi
\end{array}\right)
$$

is Hölder continuous of order $s, 0<s \leqq 1$, and strictly positive definite on $\bar{\Omega}$. The usual Gårding estimate (see [1, Theorem 7.6]) implies that defining

$$
\mathscr{B}_{0}(w)=([\Phi, w], w) \quad \text { for all } \quad w \in H_{0}^{2}(\Omega)
$$

we have

$$
\mathscr{B}_{0}(w) \geqq c_{1}\|w\|_{1,2}^{2}-c_{0}\|w\|_{2}
$$

with some positive constants $c_{0}$ and $c_{1}$.

More strongly we assume that:

$$
\mathscr{B}_{0}(w) \geqq c_{3}\|w\|_{1,2}^{2}, \quad w \in H_{0}^{2}(\Omega)
$$

with some positive constant $c_{3}$.

For completeness and as some of our results are formulated in a slightly more precise form than in $[4,2]$, we derive a number of basic properties of the von Kármán equations. 
Let us introduce the bilinear form $\mathscr{A}_{0}(u, v)=(\Delta u, \Delta v)$ for all $u, v \in H_{0}^{2}(\Omega)$. This bilinear form is continuous and the associated quadratic functional $\mathscr{A}_{0}(u)$ is positive on $H_{0}^{2}(\Omega)$.

Accordingly, we may define a linear bounded selfadjoint operator $U: H_{0}^{2}(\Omega) \rightarrow H_{0}^{2}(\Omega)$ via the theorem of Lax-Milgram (see [1, Theorem 8.11]) such that

$$
\mathscr{A}_{0}(U u, v)=(u, v)_{2,2} \quad \text { for all } \quad u, v \in H_{0}^{2}(\Omega)
$$

Since by the Poincare lemma the norm induced by $\mathscr{A}_{0}(u)$ on $H_{0}^{2}(\Omega)$ is equivalent to \|\|$_{2,2}$ the inverse $U^{-1}$ of $U$ exists and is bounded. For all $u, v, \psi \in \boldsymbol{H}_{0}^{2}(\Omega)$, we set

$$
Q(u, v, \psi)=\int_{\Omega}\left(\left(u_{y y} w_{x}-u_{x y} w_{y}\right) \psi_{x}+\left(u_{x x} w_{y}-u_{x y} w_{x}\right) \psi_{y}\right) d x
$$

Integration by parts shows that $Q(u, v, \psi)$ is a symmetric function of its variables. We use the notion of negative norm \|\|$_{-s}$ on $H_{0}^{2}(\Omega)$ due to P. Lax (see [13, III.10, Theorem 1]). Note that $f \in H_{0}^{2}(\Omega)^{*}$ thus by Riesz's theorem exists a $J f \in H_{0}^{2}(\Omega)$ such that $(J f, v)_{2,2}=(f, v)$ since $(f, v)$ is a continuous functional on $H_{0}^{2}(\Omega)$. It follows that the weak solution of the equations $\mathscr{A}_{0}(u, v)=(f, v), f \in H_{0}^{2}(\Omega)^{*}$ and all $v \in H_{0}^{2}(\Omega)$ is given by $u=U^{-1} J f \in H_{0}^{2}(\Omega)$. Now we have

$$
([u, u], v)=-Q(u, u, v) \quad \text { for all } \quad u, v \in H_{0}^{2}(\Omega)
$$

integrating by parts and, hence,

$$
|([u, u], v)| \leqq|Q(u, u, v)| \leqq \text { const }\|u\|_{2,2}\|u\|_{1,4}\|v\|_{1,4},
$$

by Hölder's inequality,

$$
\leqq \text { const }\|u\|_{2,2}^{2}\|v\|_{2,2}
$$

by Sobolev's inequality, for all $v \in H_{0}^{2}(\Omega),\|v\|_{2,2} \leqq 1$. Hence,

$$
\|[u, u]\|_{-2} \leqq \text { const }\|u\|_{2,2}^{2} \text {. }
$$

Note that equations (4.10) and (4.11) may be reformulated in the form:

$$
\begin{gathered}
\mathscr{A}_{0}(u, v)=\lambda \mathscr{B}_{0}(u, v)-\left(\left[u, U^{-1} J[u, u]\right], v\right), \quad \text { for all } v \in H_{0}^{2}(\Omega) ; \\
\phi=-U^{-1} J[u, u] .
\end{gathered}
$$

That the formulation given in (4.12) is, in fact, well defined is a consequence of the following lemma. Part (a) of the lemma refines the estimate given above. For $u \in H_{0}^{2}(\Omega)$, we set $C(u)=\left[u, U^{-1} J[u, u]\right]$ and $f(u)=\frac{1}{4}(C(u), u)$.

Lemma 4. (a) $\left\|U^{-1} J[u, u]\right\|_{2,2} \leqq$ const $\|u\|_{2,2}^{3 / 2}\|u\|_{2,2}^{1 / 2}$ for all $u \in H_{0}^{2}(\Omega)$. 
(b) $f(u) \leqq$ const $\|u\|_{2,2}^{7 / 2}\|u\|_{1,2}^{1 / 2}$ for all $u \in H_{0}^{2}(\Omega)$.

(c) $f(u) \geqq 0$ for all $u \in H_{0}^{2}(\Omega)$.

Proof. Recall that $H_{0}^{2}(\Omega) \subset H^{3 / 2,2}(\Omega)$ taken together with the Sobolev and interpolation estimates (see the references in Section 2) yield:

$$
\|u\|_{1,4} \leqq \text { const }\|u\|_{3 / 2,2} \leqq \text { const }\|u\|_{1,2}^{1 / 2}\|u\|_{2,2}^{1 / 2} .
$$

Then we have for $u, \psi \in H_{0}^{2}(\Omega)$,

$$
\begin{aligned}
\left(U^{-1} J[u, u], \psi\right)_{2,2} & =\left(J[u, u], \psi^{\prime}\right)_{2,2}, \quad \text { with } \psi^{\prime}=U^{-1} \psi, \\
& =\left([u, u], \psi^{\prime}\right) \\
& =-\left(Q\left(u, u, \psi^{\prime}\right), \quad\right. \text { by integration by parts. }
\end{aligned}
$$

It follows by Hölder's inequality and (4.13) that

$$
\begin{aligned}
\left.\mid\left(U^{-1} J[u, u]\right), \psi\right)_{2,2} \mid & \leqq \text { const }\|u\|_{2,2}\|u\|_{1,4}\left\|\psi^{\prime}\right\|_{1,4} \\
& \leqq \text { const }\|u\|_{2,2}^{3 / 2}\|u\|_{1,2}^{1 / 2}\left\|\psi^{\prime}\right\|_{2,2} \\
& \leqq \text { const }\|u\|_{2,2}^{3 / 2}\|u\|_{1,2}^{1 / 2}\|\psi\|_{2,2},
\end{aligned}
$$

for all $\psi \in H_{0}^{2}(\Omega),\|\psi\|_{2,2} \leqq 1$. We conclude that

$$
\left\|U^{-1} J[u, u]\right\|_{2,2} \leqq \text { const }\|u\|_{2,2}^{3 / 2}\|u\|_{1,2}^{1 / 2} .
$$

Now set $\phi=U^{-1} J[u, u]$. Then $f(u)=\frac{1}{4}\left([u, \phi], U^{-1} u\right)=-\frac{1}{4} Q\left(u, \phi, U^{-1} u\right)$. It follows that

by Sobolev's inequality

$$
\begin{aligned}
f(u) & \leqq \text { const }\|u\|_{2,2}\|\phi\|_{1,4}\left\|U^{-1} u\right\|_{1,4} \\
& \leqq \text { const }\|u\|_{2,2}^{2}\|\phi\|_{1,4},
\end{aligned}
$$

$$
\leqq \text { const }\|u\|_{2,2}^{7 / 2}\|u\|_{1,2}^{1 / 2}
$$

by part (a).

Finally, note that

$$
f(u)=-\frac{1}{4} Q(u, \phi, u)=\frac{1}{4}\left(\phi,\left[\left(u_{y y} u_{x}-u_{x y} u_{y}\right)_{x}+\left(u_{x x} u_{y}-u_{x y} u_{y}\right)_{y}\right),\right.
$$

by symmetry and integration by parts,

$$
=\frac{1}{4}(\phi,[u, u])
$$




$$
\begin{aligned}
& =\frac{1}{4}\left(U^{-1} J[u, u],[u, u]\right) \\
& =\frac{1}{4}\left([u, u], U^{-1} J[u, u]\right) \\
& =\frac{1}{4}\left(J[u, u], U^{-1} J[u, u]\right)_{2,2} \\
& =\frac{1}{4}\left\|U^{-1 / 2} J[u, u]\right\|_{2,2}^{2} \geqq 0, \quad \text { for all } u \in H_{0}^{2}(\Omega) .
\end{aligned}
$$

Lemma 5. (a) The operator $C(u)$ is completely continuous from $H_{0}^{2}(\Omega)$ to $H_{0}^{2}(\Omega)^{*}$ with norm $\|C(u)\|_{-2} \leqq$ const $\|u\|_{2,2}^{5 / 2}\|u\|_{1,2}^{1 / 2}$ for all $u \in H_{0}^{2}(\Omega)$.

(b) The functional $f(u)$ is weakly sequentially continuous on $H_{0}^{2}(\Omega)$.

Proof. Suppose that $u_{n} \stackrel{m}{\rightarrow}$ in $H_{0}^{2}(\Omega)$. Then $\sup _{n}\left\|u_{n}\right\|_{2,2}<\infty$.

We first show that $U^{-1} J[u, u]$ is completely continuous. One has as before

$$
\begin{aligned}
\left(J[u, u]-J\left[u_{n}, u_{n}\right], U^{-1} \psi\right)_{2,2} & =\left([u, u]-\left[u_{n}, u_{n}\right], U^{-1} \psi\right) \\
& =\left(Q\left(u, u, \psi^{\prime}\right)-Q\left(u_{n}, u_{n}, \psi^{\prime}\right)\right) .
\end{aligned}
$$

It is readily seen that

$$
\begin{aligned}
\left|Q\left(u, u, \psi^{\prime}\right)-Q\left(u_{n}, u_{n}, \psi^{\prime}\right)\right| \leqq & \left|Q\left(u, u, \psi^{\prime}\right)-Q\left(u_{n}, u, \psi^{\prime}\right)\right| \\
& +\left|Q\left(u_{n}, u, \psi^{\prime}\right)-Q\left(u_{n}, u_{n}, \psi^{\prime}\right)\right| \\
= & \left|Q\left(\psi^{\prime}, u, u\right)-Q\left(\psi^{\prime}, u, u_{n}\right)\right| \\
& +\left|Q\left(u_{n}, u, \psi^{\prime}\right)-Q\left(u_{n}, u_{n}, \psi^{\prime}\right)\right|
\end{aligned}
$$

by the symmetry of $Q$,

$$
\leqq \text { const }\left\{\|u\|_{2,2}+\sup _{n}\left\|u_{n}\right\|_{2,2}\right\}\left\|u-u_{n}\right\|_{1,4}\|\psi\|_{2,2} \rightarrow 0 \quad \text { as } \quad n \rightarrow \infty
$$

for all $\psi \in H_{0}^{2}(\Omega),\|\psi\| \leqq 1$, using the fact that $H_{0}^{2}(\Omega)$ is embedded in $H^{1,4}(\Omega)$ continuously.

It follows that

$$
\left\|U^{-1} J[u, u]-U^{-1} J\left[u_{n}, u_{n}\right]\right\|_{2,2} \rightarrow 0 \quad \text { as } n \rightarrow \infty
$$

The estimate

$$
\begin{aligned}
\mid(C(u), \psi \mid & =|Q(u, \phi, \psi)| \\
& \leqq \text { const }\|u\|_{2.2}\|\phi\|_{1,4}\|\psi\|_{1,4}
\end{aligned}
$$




$$
\begin{aligned}
& \leqq \text { const }\|u\|_{2,2}\|\phi\|_{2,2}\|\psi\|_{2,2} \\
& \leqq \text { const }\|u\|_{2,2}^{5 / 2}\|u\|_{1,2}^{1 / 2}\|\psi\|_{2,2}
\end{aligned}
$$

for all $\psi \in H_{0}^{2}(\Omega),\|\psi\|_{2,2} \leqq 1$, implies that $C(u) \in H_{0}^{2}(\Omega)^{*}$, with negative norm $\|C(u)\|_{-2} \leqq$ const $\|u\|_{2,2}^{5 / 2}\|u\|_{1,2}^{1 / 2}$. Also

$$
\begin{aligned}
\left|\left(C(u)-C\left(u_{n}\right), \psi\right)\right| \leqq & |Q(\psi, \phi, u)|-Q\left(\psi, \phi, u_{n}\right) \mid \\
& +\left|Q\left(u_{n}, \phi, \psi\right)-Q\left(u_{n}, \phi_{n}, \psi\right)\right|
\end{aligned}
$$

as above, by symmetry, so that,

$$
\begin{aligned}
& \leqq \text { const }\left(\|\psi\|_{2,2}\|\phi\|_{1,4}\left\|u-u_{n}\right\|_{1,4}+\left\|u_{n}\right\|_{2,2}\left\|\phi-\phi_{n}\right\|_{1,4}\|\psi\|_{1,4}\right) \\
& \leqq \text { const }\left\{\|u\|_{2,2}^{2}\left\|u-u_{n}\right\|_{1,4}+\sup \left\|u_{n}\right\|_{2,2}\left\|\phi-\phi_{n}\right\|_{1,4}\right\}\|\psi\|_{2,2}
\end{aligned}
$$

for all $\|\psi\|_{2,2} \leqq 1$

It follows that

$$
\left\|C(u)-C\left(u_{n}\right)\right\|_{-2} \leqq \text { const }\left(\left\|u-u_{n}\right\|_{1,4}+\left\|\phi-\phi_{n}\right\|_{2,2}\right) \rightarrow 0
$$

as $n \rightarrow \infty$, by (4.14) and the fact that $H_{0}^{2}(\Omega)$ is compactly embedded in $H^{1.4}(\Omega)$.

Finally since $f(u)=\frac{1}{4}(C(u), u)$ and $C\left(u_{n}\right)^{\stackrel{s}{\rightarrow}} C(u)$ in $H_{0}^{2}(\Omega)^{*}$ if $u_{n} \stackrel{w}{\rightarrow} u$ in $H_{0}^{2}(\Omega)$, it is immediate that $f\left(u_{n}\right) \rightarrow f(u)$.

Observation. Conditions (i), (ii) and (iv) of Theorem 6.6.1 in [4] are obviously satisfied. If $u_{n} \stackrel{\mathfrak{m}}{\rightarrow} u$ in $H_{0}^{2}(\Omega)$ and $\mathscr{A}^{\prime}\left(u_{n}\right) \stackrel{s}{\rightarrow} \mathscr{A}^{\prime}(u)$ in $H_{0}^{2}(\Omega)^{*}$ then $\mathscr{A}^{\prime}\left(u_{n}\right) \cdot u_{n} \rightarrow \mathscr{A}^{\prime}(u) . u$. However, by Lemma $5\left(\right.$ a) $f^{\prime}\left(u_{n}\right) \stackrel{s}{\rightarrow} f^{\prime}(u)$ in $H_{0}^{2}(\Omega)^{*}$ so that $A_{0} u_{n} s A_{0}(u)$ in $H_{0}^{2}(\Omega)^{*}$ and, hence, $\mathscr{A}\left(u_{n}\right) \rightarrow \mathscr{A}_{0}(u)$. This implies that $\lim _{n \rightarrow \infty}\left\|u_{n}\right\|_{2,2}^{2}=\|u\|_{2,2}^{2}$. It follows that $u_{u} \stackrel{s}{\rightarrow} u$ in $H_{0}^{2}(\Omega)$. By the weak sequential continuity of $f(u), f\left(u_{n}\right) \rightarrow f(u)$ and we conclude that $\mathscr{A}\left(u_{n}\right) \rightarrow \mathscr{A}(u)$ as $n \rightarrow \infty$. It follows that condition (iii) of Theorem 6.6.1 in [4] is satisfied.

It follows from (4.12) that we may formulate the Liusternik-Schnirelman theory with respect to the pair of functionals $\left(\mathscr{A}(u), \mathscr{B}_{0}(u)\right)$, where $\mathscr{A}(u)=\frac{1}{2} \mathscr{A}_{0}(u)+f(u)$ at level $M_{c}=\{u: \mathscr{A}(u)=c\}$.

As before the map $\tilde{\tau}(u)=\tau(u) u$ is an odd radial one-one onto map of $\Sigma_{c}$ into $M_{c}$, where

$$
\tau(u)=\frac{\sqrt{2}}{\sqrt{1+\left(1+\frac{4 f(u)}{c}\right)^{1 / 2}}}
$$

is the solution of the equation $\tau^{2} c+\tau^{4} f(u)=c$. In fact, this map is the inverse of 


$$
\hat{\tau}: u \rightarrow \frac{u}{\sqrt{1-\frac{f(u)}{c}}}
$$

of $M_{c} \rightarrow \Sigma_{c}$. It follows that $\tilde{\tau}$ is a homeomorphism since $\hat{\tau}$ is a homeomorphism by the same argument as given in Section 4.1.

Theorem 2'. For the von Kármán equations for buckled plates under the hypotheses $H^{\prime}$ the asymptotic distribution of eigenvalues $v(t)$ is given by

$$
\left.v(t)=g \delta t+0^{(2-(s / s+1)) / 2}\right) \quad \text { as } \quad t \rightarrow \infty
$$

at any level $M_{c}, c>0$.

Proof. First,

$$
\begin{aligned}
c_{t}^{0} & =\sup _{V \in S_{t}} \inf _{u \in V \subset \Sigma_{c}}\left(\frac{1}{2} \mathscr{B}_{0}(u)\right) \\
& \leqq \sup _{V \in S_{t}} \inf _{u \in V \subset \Sigma_{c}} \frac{1+\sqrt{1+\frac{4 f(u)}{c}} \frac{\mathscr{B}_{0}(\tilde{\tau}(u)}{2}}{2} \\
& \leqq \sup _{V \in S_{t}} \inf _{w \in V \subset M_{c}}\left(\left(1+\frac{f(u)}{c}\right) \frac{\mathscr{B}_{0}(w)}{2}\right), \quad w=\tilde{\tau}(u),
\end{aligned}
$$

by the invariance of the class $S_{t}$ under the homeomorphism $\tilde{\tau}$. Hence,

$$
c_{t}^{0} \leqq \sup _{V \in S_{t}} \inf _{w \in V \subset M_{c}}\left(\left(1+K_{1}(c)\|u\|_{1,2}^{1 / 2}\right) \frac{\mathscr{B}_{0}(w)}{2}\right)
$$

using Lemma $4(\mathrm{~b})$ and the estimate $\|u\|_{2,2} \leqq$ const $c^{1 / 2}$ for $u \in \Sigma_{\mathbf{c}}$.

Also, we have the inequality

$$
\tau(u)^{-1} \leqq \sqrt{1+\left(1+\frac{4 f(u)^{1 / 2}}{c}\right)} / \sqrt{2} \leqq K_{2}(c),
$$

by Lemma $4(\mathrm{~b})$, and $\|u\|_{2,2} \leqq$ const $c^{1 / 2}$ for $u \in \Sigma_{c}$. From (4.15) and (4.16) we conclude that

$$
c_{t}^{0} \leqq \sup _{V \in S_{t}} \inf _{w \in V \subset M_{c}}\left(\left(1+K_{3}(c)\|w\|_{1,2}\right) \frac{\mathscr{B}_{0}(w)}{2}\right)
$$


and by $H^{\prime}(b)$

$$
\begin{aligned}
c_{t}^{0} & \leqq \sup _{V \in S_{t}} \inf _{w \in V \in M_{c}}\left(1+K_{4}(c) \mathscr{B}_{0}(w)^{1 / 4}\right) \frac{\mathscr{B}_{0}(w)}{2} \\
& =c_{t}+O\left(\left(c_{t}\right)^{5 / 4}\right) \quad \text { as } \quad t \rightarrow \infty .
\end{aligned}
$$

On the other hand, an argument similar to that given in Theorem 2 shows that

$$
c_{t} \leqq c_{t}^{0} \quad \text { as } \quad t \rightarrow \infty
$$

From (4.18) and (4.19) we conclude that

$$
c_{t}=c_{t}^{0}+O\left(\left(c_{t}^{0}\right)^{5 / 4}\right) \quad \text { as } \quad t \rightarrow \infty \quad \text { at level } M_{c} .
$$

Finally, we observe that

$$
\lambda_{t}^{-1}=\frac{2 \mathscr{B}_{0}\left(u_{t}\right)}{\left(\mathscr{A}^{\prime}\left(u_{t}\right) \cdot u_{t}\right)}=\frac{2 c_{t}}{\left(\mathscr{A}^{\prime}\left(u_{t}\right) \cdot u_{t}\right)}, \quad u_{t} \in M_{c}
$$

One has $\left(\mathscr{A}^{\prime}\left(u_{t}\right) \cdot u_{t}\right)=\mathscr{A}_{0}\left(u_{t}\right)+\left(C\left(u_{t}\right) \cdot u_{t}\right)$ and by Lemma $5($ a)

$$
\begin{aligned}
\left|\left(C\left(u_{t}\right) \cdot u_{t}\right)\right| & \leqq \text { const }\left\|u_{t}\right\|_{2,2}^{7 / 2}\left\|u_{t}\right\|_{1,2}^{1 / 2} \\
& \leqq \text { const }\left\|u_{t}\right\|_{2,2}^{7 / 2} \mathscr{B}_{0}\left(u_{t}\right)^{1 / 4},
\end{aligned}
$$

by the coercivity of $\mathscr{A}_{0}(u)$, the positivity of $f(u)$ and $u_{t} \in M_{\mathrm{c}}$ together with $\mathrm{H}^{\prime}(\mathrm{b})$.

From (4.20), (4.21) and (4.22) it follows that

$$
\begin{aligned}
\lambda_{t}^{-1} & =2 c_{t}^{0}\left(2 c+O\left(c_{t}^{0}\right)^{1 / 4}\right)^{-1}\left(1+O\left(\left(c_{t}^{0}\right)^{1 / 4}\right)\right. \\
& =c^{-1} c_{t}^{0}+O\left(\left(c_{t}^{0}\right)^{5 / 4}\right) \quad \text { as } \quad t \rightarrow \infty
\end{aligned}
$$

Recalling that $\lambda_{t}^{0-1}=c^{-1} c_{t}^{0}$, we conclude from (4.23) that

$$
\lambda_{t}^{-1}=\left(\lambda_{t}^{0}\right)^{-1}+0\left(\left(\lambda_{t}^{0}\right)^{-5 / 4}\right) \quad \text { as } \quad t \rightarrow \infty
$$

at level $M_{c}$. The conclusion of the theorem follows immediately from (4.24) and (3.1) since

$$
c_{t}^{0}=c(g \delta) t^{-1}+O\left(t^{-(1+(s / 2(s+1)))}\right) \quad \text { as } \quad t \rightarrow \infty
$$

and

$$
\left(\lambda_{t}^{0}\right)^{-5 / 4}=0\left(t^{-(1+(s / 2(s+1))}\right), \quad 0<s \leqq 1, \quad \text { as } \quad t \rightarrow \infty
$$


Observation. The assumption made in Theorem 6.7.16 of [4] where it is shown $\lambda_{n} \geqq 0, \lambda_{n} \rightarrow \infty$ as $n \rightarrow \infty$ is equivalent in our notation to $\left(B_{0} u, u\right)>0$, by compactness this hypothesis implies $\mathrm{H}^{\prime}(\mathrm{b})$. Bifurcation type results are given in $[2,3]$.

\section{REFERENCES}

1. S. Agmon, Lectures on Elliptic Boundary Value Problems (Van Nostrand, Princeton, N.J., 1965).

2. M. Berger, A bifurcation theory for nonlinear differential equations and related topics, in: Bifurcation Theory and Nonlinear Eigenvalue Problems (Edited by J. B. Keller and S. Antman, Benjamin, New York, 1969), 113-216.

3. M. Berger, Multiple solutions of nonlinear operator equations arising from the calculus of variations, Proc. Sympos. A.M.S. 18 (1970), 10-27.

4. M. Berger, Nonlinearity and Functional Analysis (Academic Press, New York, 1977).

5. F. E. Browder, Existence theorems for nonlinear partial differential equations, Proc. Sympos. Pure Math. 16 (1970), 1-61.

6. F. E. Browder, Nonlinear eigenvalue problems and group invariance, in: Functional Analysis and Related Fields (Edited by F. E. Browder, Springer-Verlag, 1970), 1-58.

7. R. Chiappinelul, The asymptotic distribution of eigenvalues of a nonlinear problem, Boll. Un. Mat. Ital. B(6) 1 (1982), 1131-1149.

8. R. Chiappinelli, On the eigenvalues and the spectrum for a class of semilinear elliptic operators, Boll. Un. Mat. Ital. B(6) 4 (1985), 867-882.

9. S. FuCik, J. NeCas, J. Soucek and V. Soucek, Spectral Analysis of Nonlinear Operators, (Lecture Notes in Mathematics 346 Springer-Verlag, Berlin, 1973).

10. G. Metivier, Valeurs propres des problems aux limites elliptiques irregulières, Bull. Soc. Math. France Memoire 51-52 (1977), 125-219.

11. V. B. Moscatelli and M. Thompson, Asymptotic eigenvalue distributions for pairs of bilinear forms, Ricerche Mat. 32 (1983), 41-63.

12. H. TRIebel, Interpolation Theory, Function Spaces, Differential Operators (North-Holland Publishing Co., New York, 1978).

13. K. YosidA, Functional Analysis (Springer-Verlag, Berlin, 1966).

Instituto Di Matematica

UNIVERSITẢ CP193

Via Arnesano

73100 LECCE

ITALY
Instituto De Matemática

UFRGS

Campus Do Vale

9500 Bento Gonçalves

91500 Porto Alegre

Brazil 